\title{
Small bowel adenocarcinomas in celiac disease follow the CIM-MSI pathway
}

\author{
FRANK BERGMANN $^{1}$, SANDHYA SINGH ${ }^{*}$, SARA MICHEL $^{2}$, CHRISTOPH KAHLERT $^{3}$, \\ PETER SCHIRMACHER ${ }^{1}$, BURKHARD HELMKE ${ }^{4}$, MAGNUS VON KNEBEL DOEBERITZ ${ }^{2}$, \\ MATTHIAS KLOOR $^{2}$ and HENDRIK BLÄKER ${ }^{1}$
}

Departments of ${ }^{1}$ General Pathology, ${ }^{2}$ Applied Tumor Biology, Institute of Pathology, INF 220, and ${ }^{3}$ Department of Surgery, INF 110, University of Heidelberg, INF 220, 69120 Heidelberg; ${ }^{4}$ Elbe Kliniken Stade, 21682 Stade, Germany

Received August 6, 2010; Accepted August 31, 2010

DOI: 10.3892/or_00001015

\begin{abstract}
Celiac disease (CD) is an inflammatory disorder associated with an increased risk of small bowel adenocarcinoma. Recent studies have demonstrated aberrant $\mathrm{CpG}$ island methylation (CIM) in chronic inflammation, aging and cancer. We hypothesized that CIM may link CD to small bowel carcinogenesis. We determined microsatellite instability (MSI), CIM, and expression of MLH1 and MGMT in 3 CDassociated small bowel carcinomas and corresponding nonneoplastic mucosa. The results were compared to those of small bowel mucosa from CD patients without carcinoma and 20 small bowel carcinomas from a non-CD origin. A high level CIM/MSI phenotype was found in all of the $3 \mathrm{CD}$ associated carcinomas and was associated with loss of MLH1 expression due to hypermethylation of the $M L H 1$ promoter. This phenotype was noted in only 2 of the 20 investigated non-CD-associated carcinomas. Low-level CIM was already detectable in 9 of the 12 non-neoplastic mucosa samples of $\mathrm{CD}$ patients and in non-CD-associated carcinomas of elderly patients. In conclusion, our data reveal that the high-level CIM/MSI pathway is typical of CD-associated small bowel carcinomas and indicate that aberrant $\mathrm{CpG}$ island methylation links CD and carcinogenesis. The data further suggest that CD should be considered in patients with small bowel adenocarcinoma, particularly when the tumors display MSI.
\end{abstract}

\section{Introduction}

Celiac disease (CD) is an inflammatory disorder caused by intolerance to gluten. Previously considered a disease of early

Correspondence to: Dr Hendrik Bläker, Department of Pathology, University of Heidelberg, INF 220, 69120 Heidelberg, Germany

E-mail: hendrik_blaeker@med.uni-heidelberg.de

*Contributed equally

Key words: epigenetics, small intestine, celiac disease, cancer infancy, late age of clinical onset and diagnosis is currently well recognized $(1,2)$. In CD patients, exposure to gluten results in chronic inflammation of the small bowel mucosa characterized by intraepithelial lymphocytosis, crypt hyperplasia and villous atrophy $(3,4)$. CD is a well-established risk factor for small bowel lymphoma (5-7). In several recent studies on CD patients, the association with small bowel adenocarcinomas (SBAs) was also investigated, and a relative risk of 10- to 80-fold for this tumor type was reported for CD $(6,8,9)$. Given the comparatively high, yet not precisely determined, prevalence of $\mathrm{CD}$ in various populations $(0.15$ $2.6 \%$ ) (10), one would expect a significant share of CDassociated SBAs in a larger collection of these tumors. The results of two studies concerning this issue, however, are conflicting. While Howdle et al (9) reported 13\% of SBAs collected in a nation-wide survey in the UK to be CDassociated (9), no signs of CD were reported among 69 SBAs collected in Northern Ireland, an area of high CD prevalence (11).

The association of gluten-induced chronic inflammation with small bowel tumorigenesis is reminiscent of colon cancer in inflammatory bowel disease. Aberrant methylation at $\mathrm{CpG}$ islands (CIM) is an epigenetic phenomenon originally described in cancer $(12,13)$, but CIM has also previously been reported in association with non-neoplastic conditions including aging and chronic inflammation (14-16). Since CIM can contribute to carcinogenesis through transcriptional silencing of tumor-suppressor genes, it may provide an explanation for the well-known association of aging and chronic inflammation with tumor risk.

We investigated a large cohort of consecutively collected SBAs for the presence of CD and analyzed the molecular pathogenesis of $\mathrm{CD}$-associated carcinomas in comparison to non-CD-associated carcinomas. Herein, we showed that in contrast to non-CD-associated SBAs, all CD-associated carcinomas followed a molecular pathway characterized by high-level CIM, MLHI inactivation and microsatellite instability (MSI). Our data indicate CD-associated carcinogenesis to be a sequential process preceded by CIM, initiated by methylation-induced $M L H 1$ silencing and promoted by MSI. 


\section{Materials and methods}

A consecutive cohort of 70 primary SBAs collected over a time-span of 20 years were retrieved from our Institute of Pathology. Histologic sections were reviewed for the presence of CD. Diagnosis of CD was based on CD typical morphology of non-neoplastic mucosa and on clinical information. Three patients with $\mathrm{CD}$-associated SBAs were identified. In one patient $C D$ was diagnosed in early childhood; in the other two cases, CD was diagnosed at the time of tumor resection. In one case a small bowel resection for anaplastic T-cell lymphoma was performed 4 years before small bowel carcinoma resection; in the other 2 cases, no synchronous or metachronous tumors occurred.

Twenty non-CD-associated carcinomas previously analysed for MSI (17) [4 MSI-H, 16 microsatellite stable (MSS)], non-neoplastic mucosa of 5 non-CD carcinoma cases as well as 9 biopsy specimens of CD patients without carcinoma were chosen for the comparative study of methylation. Clinical data for the individual cases is documented in Table I together with the results of methylation and MSI analysis. The study was approved by the Institutional Ethics Committee (application no. 206/05) of the Medical Faculty of Heidelberg University.

DNA isolation. For isolation of DNA from tumor and nonneoplastic mucosa, tissue slides $(5-\mu \mathrm{m})$ were prepared. Tumor tissue and normal mucosa were isolated by manual microdissection of unstained slides. DNA from non-neoplastic mucosa was taken from the resection margins whenever possible ( 2 of the $3 \mathrm{CD}$-associated SBAs and in 4 of 5 nonCD-associated SBAs) or was taken at least a distance of $0.5 \mathrm{~cm}$ from the tumor. DNA was prepared using the DNeasy tissue kit (Qiagen, Hilden, Germany) according to the manufacturer's instructions.

MSI analysis. Tumor samples were typed for MSI using the standard NCI/ICG-HNPCC marker panel (18) and CAT25 as described previously (19).

Methylation-specific PCR. Methylation-specific (MSP) PCR was performed for $5 \mathrm{CpG}$ islands known to be methylated in colorectal cancer: MINT1, MINT2, MGMT, MLH1 and p16 $(C D K N 2 A)$. Primers and PCR conditions were used as described previously [MINT1 and MINT2 (20); MGMT (21); p16 (22); MLH1 (23)]. DNA was bisulfite-converted using the EZ DNA Methylation-Gold kit (Zymo Research, USA) according to the manufacturer's instructions. One-fifth of a conversion reaction was used as template.

PCR products were visualized on a $2 \%$ agarose TAE gel. Methylation status was assessed based on the presence or absence of amplified product in the MSP PCR. CIM-high, CIM-low and CIM-negative were scored when more than $50 \%, 20-50 \%$ and $0 \%$ of the investigated loci were positive for MSP PCR, respectively.

Immunohistochemistry. To compare the methylation status of MLH1 and MGMT with protein expression, immunohistochemistry was performed on $5-\mu \mathrm{m}$ paraffin sections. For the mismatch repair protein MLH1 (clone G168-15, BD
Pharmingen, Heidelberg, Germany; dilution 1:100) and MGMT (clone MT3.1, Thermo Scientific, Fremont, CA, USA; dilution 1:30) slides were pre-treated with 10-min microwave boiling in Dako target retrieval buffer, $\mathrm{pH} 9.0$ (Dako, Hamburg, Germany). The antibody was incubated for $30 \mathrm{~min}$ at room temperature. For visualization purposes, the labeled immunoperoxidase method with AEC as a chromogen was applied (Dako). In non-neoplastic mucosa, loss of MGMT expression was scored when all cells within a crypt or groups of crypts were negative for nuclear staining while stromal cells were positive. In tumors, loss of MGMT and MLH1 expression was scored when all tumor cells were negative while the stromal cells and adjacent non-neoplastic mucosa were positive.

\section{Results}

Microsatellite instability in $C D$ - and non-CD-associated SBAs. MSI was detected in all of the $3 \mathrm{CD}$-associated adenocarcinomas. Among the 20 non-CD-associated adenocarcinomas used for the comparative study of methylation, MSI-H was noted in 4 tumors. All three CD-associated adenocarcinomas displayed loss of MLH1 expression (Fig. 1), and all of the 4 MSI-H carcinomas in the non-CD carcinoma cohort were MLH1-negative.

CpG island methylation in SBAs and non-neoplastic mucosa of patients with and without CD. All 3 CD-associated SBAs and 5 of the 20 non-CD-associated SBAs were CIM-high. Eight of the 20 non-CD-associated SBA were CIM-low, and 7 of 20 were CIM-negative. CIM was related to patient age. While the average age of the patients with CIM-negative carcinomas was 55 years, it was 73 years for patients showing CIM. CIM-high with MLH1 methylation and loss of MLH1 expression was found in all 3 of the CD-associated SBAs and in 2 of the 4 MSI-H non-CD-associated SBA. CIM-high without MLH1 methylation was found in 3 MSS SBAs. CIMlow with MLH1 methylation was noted in 2 of the MSI-H non-CD-associated carcinomas. The results of the methylation analyses in individual cases and at the tested loci are summarized in Table I.

Non-neoplastic mucosa. CIM-low was identified in the nonneoplastic mucosa of all of the CD-associated SBA cases (Table I, Fig. 1). CIM-low was also identified in 6 of the 9 CD mucosa samples not associated with SBAs as well as in 4 of the 5 non-neoplastic mucosa samples of the non-CDassociated carcinomas. No methylation of $M L H 1$ or $C D K N 2 A$ was noted in the non-neoplastic mucosa specimens. MINT1 was methylated in 5 of the $9 \mathrm{CD}$ mucosa samples and in 3 of the 5 non-CD mucosa samples. MINT2 methylation was found in $2 \mathrm{CD}$ and in 2 non-CD mucosa samples. MGMT methylation was observed in 1 non-CD mucosa sample.

Except for case 401, where $M G M T$ methylation was exclusively found in non-neoplastic mucosa, methylation of a specific locus in non-neoplastic mucosa was also found in the corresponding tumor tissue (Table I).

Expression of MLH1 and MGMT. Loss of MLH1 expression was found in all of the $3 \mathrm{CD}$-associated carcinomas and it 
Table I. Clinical data, tumor location, methylation and microsatellite status of the investigated carcinomas.

\begin{tabular}{|c|c|c|c|c|c|c|c|c|}
\hline Case & Location & Age/gender & MGMT & MINT1 & MINT2 & P16 & MLH1 & MSI/MSS \\
\hline \multicolumn{9}{|c|}{ Non-neoplastic mucosa and tumors in celiac disease } \\
\hline \multicolumn{9}{|l|}{ N884 } \\
\hline $\mathrm{T} 884$ & Duo & 47/female & & & & & & MSI-H \\
\hline \multicolumn{9}{|l|}{ N418 } \\
\hline $\mathrm{T} 418$ & Jej/lle & 57/male & & & & & & MSI-H \\
\hline \multicolumn{9}{|l|}{ N332 } \\
\hline T332 & Jej/lle & $59 / \mathrm{male}$ & & & & & & MSI-H \\
\hline \multirow{2}{*}{\multicolumn{9}{|c|}{ Non-neoplastic mucosa and tumors without celiac disease }} \\
\hline \multicolumn{8}{|c|}{ 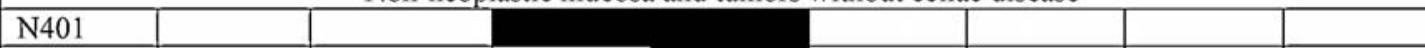 } & \\
\hline $\mathrm{T} 401$ & Jej/Ile & $59 / \mathrm{male}$ & & & & & & MSS \\
\hline \multicolumn{9}{|l|}{ N174 } \\
\hline $\mathrm{T} 174$ & Duo & $61 /$ male & & & & & & MSS \\
\hline \multicolumn{9}{|l|}{ N180 } \\
\hline$T 180$ & Duo & $64 / \mathrm{male}$ & & & & & & MSS \\
\hline \multicolumn{9}{|l|}{ N343 } \\
\hline T343 & Ile & $83 /$ male & & & & & & MSS \\
\hline \multicolumn{9}{|l|}{ N376 } \\
\hline $\mathrm{T} 376$ & Jej/lle & $81 /$ female & & & & & & MSI-H \\
\hline \multicolumn{9}{|c|}{ Tumors without celiac disease } \\
\hline $\mathrm{T} 410$ & Duo & 74/female & & & & & & MSI-H \\
\hline T172 & Ile & $84 /$ male & & & & & & MSI-H \\
\hline T361 & Ile & $71 /$ male & & & & & & MSS \\
\hline T656 & Jej/lle & $75 /$ male & & & & & & MSS \\
\hline $\mathrm{T} 820$ & Ile & 72/female & & & & & & MSS \\
\hline T970 & Duo & $74 / \mathrm{male}$ & & & & & & MSS \\
\hline T249 & Ile & $80 /$ female & & & & & & MSS \\
\hline $\mathrm{T} 303$ & Duo & $76 /$ male & & & & & & MSS \\
\hline T246 & Ile & 29/male & & & & & & MSS \\
\hline $\mathrm{T} 502$ & Jej/Ile & $30 /$ male & & & & & & MSS \\
\hline T208 & Duo & 54/female & & & & & & MSS \\
\hline $\mathrm{T} 235$ & Jej/Ile & $68 / \mathrm{female}$ & & & & & & MSS \\
\hline $\mathrm{T} 486$ & Ile & 69/female & & & & & & MSS \\
\hline T195 & Duo & $76 /$ male & & & & & & MSS \\
\hline \multicolumn{3}{|c|}{ Marsh } & \multicolumn{6}{|c|}{ Non-neoplastic mucosa in celiac disease } \\
\hline CD142 & II-III & $62 /$ male & & & & & & \\
\hline CD743 & 0 & 62/female & & & & & & \\
\hline CD359 & III & 53/female & & & & & & \\
\hline CD505 & III & $45 /$ female & & & & & & \\
\hline CD507 & III & 46/female & & & & & & \\
\hline CD418 & III & $72 /$ male & & & & & & \\
\hline CD271 & III & 46/female & & & & & & \\
\hline CD417 & 0 & 40/female & & & & & & \\
\hline CD413 & I & $45 / \mathrm{male}$ & & & & & & \\
\hline
\end{tabular}

Black squares, methylation; blank squares, no methylation; grey squares, not determined.

correlated well with a strong PCR signal for the methylated DNA at the $M L H I$ promoter while the corresponding PCR signal for non-methylated DNA was faint or absent (Fig. 1). MLH1 expression was retained in the surrounding nonneoplastic mucosa of the CD cases where no MLH1 methylation was found. Loss of MLH1 expression together with MLH1 methylation was present in all 4 MSI-H non-CD carcinomas (17). MGMT methylation correlated with loss of MGMT expression in the CD-associated SBAs and in the non-neoplastic mucosa of CD case 418 (Fig. 1). Loss of MGMT expression was noted in 2 non-CD carcinomas and methylation of MGMT was found in both (T361, T343). Five
non-CD carcinomas with $M G M T$ methylation showed retained expression of MGMT indicating incomplete $M G M T$ silencing by promoter methylation.

\section{Discussion}

Among a cohort of 70 consecutively collected small bowel adenocarcinomas, we identified 3 cases associated with CD $(4.3 \%)$. Given a CD prevalence of $0.5 \%$ in the German population (10) our data are in line with a nation-wide survey in the UK (9), where CD is approximately twice as common as in Germany (24). In this study, Howdle et al (9) reported 


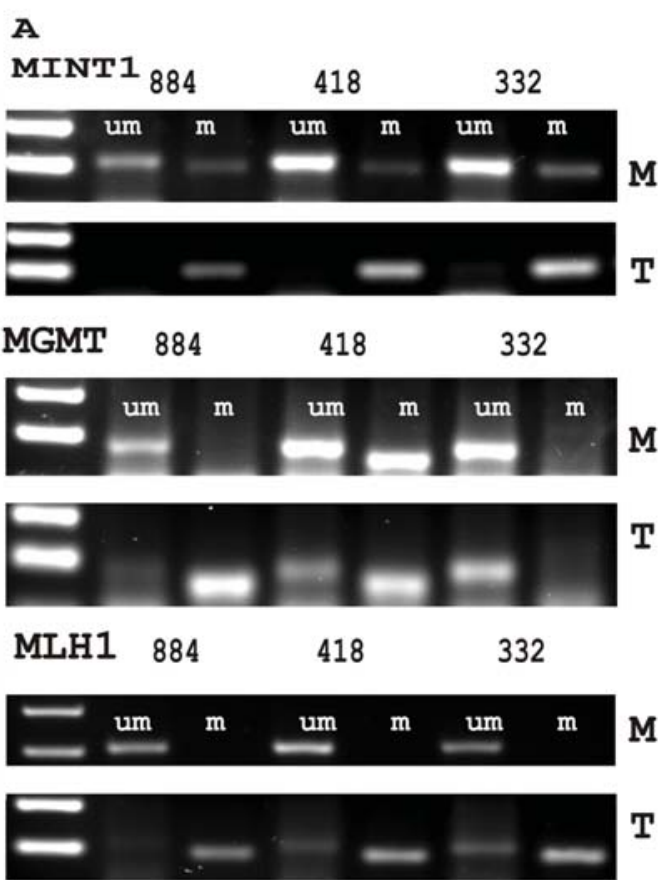

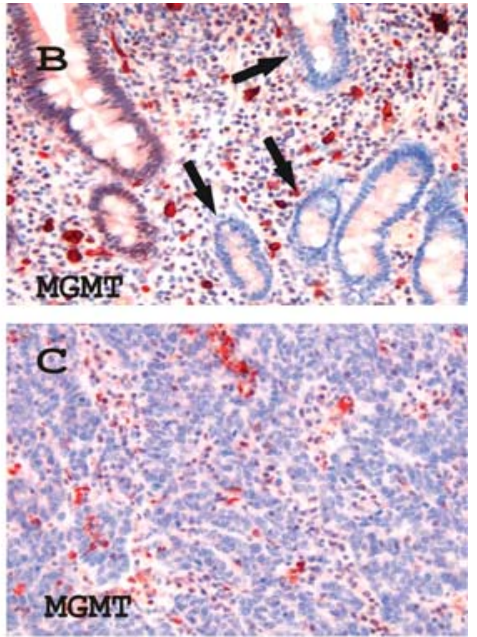

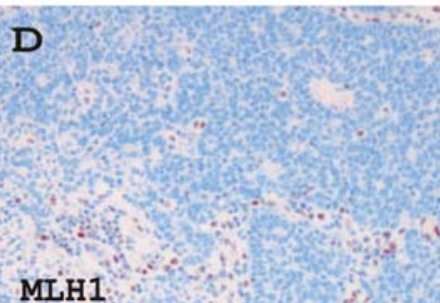

Figure 1. Methylation analysis of CD-associated carcinomas (T) and corresponding non-neoplastic mucosa (M) for MINT1, MGMT and MLH1 (A). Methylation (m) of MINT1 is noted in all of the cases of non-neopolastic mucosa and methylation of MGMT in the non-neoplastic mucosa of 418. No MLH1 methylation is noted in the non-neoplastic mucosa but in all of the tumors with a very faint amplification of the unmethylated allele indicating biallelic methylation. Methylation of MGMT correlates with loss of protein expression in singular crypts of the non-neoplastic mucosa (N418; B, arrows). Methylation of MGMT and MLH1 correlate with complete loss of MGMT (C) and MLH1 (D) expression in the tumor cells while stromal cells and lymphocytes retain protein expression (T418; C and D).

13\% CD-associated tumors among 169 primary small bowel adenocarcinomas. However, in a similar study in Northern Ireland, in which pathology reports were reviewed for villous atrophy, CD was identified in $18 \%$ of small bowel lymphomas but in none of the 69 adenocarcinomas. The reason for this disparity is not clear, but some explanations should be considered. On the one hand, the infiltrative and hyperplastic types of CD (MARSH types 1 and 2) lack significant changes in villous architecture. Therefore, absence of villous atrophy does not rule out $\mathrm{CD}$. On the other hand, CD's association with SBA is less known to pathologists and clinicians than its association with lymphoma, the enteropathy-type lymphoma in particular. There is no tumor entity defined as enteropathy type-associated adenocarcinoma. When assessing a resection specimen of small intestinal adenocarcinoma, subtile inflammatory alterations of $\mathrm{CD}$ may remain unrecognized by the pathologist.

Diagnosis of CD in SBA is of clinical importance in order to avoid misinterpretation of postoperative malabsorption and to prevent $\mathrm{CD}$-associated complications by a gluten-free diet (25). The small bowel lymphoma risk in CD significantly decreases with successful treatment (26). This may not be the case for the risk of SBA in CD. Kingham et al (27) reported the occurrence of CD-associated SBA despite the remission of $\mathrm{CD}$ under a gluten-free diet. Corroborating this finding, one of the patients presented herein developed SBA although $\mathrm{CD}$ diagnosed in early childhood was successfully treated.
SBA is rare (incidence rate 2, 3/100,000, SEER), and compared to colorectal cancer its occurrence is more likely to be related to a cancer-prone condition. Apart from $\mathrm{CD}$ and Crohns's disease, FAP and HNPCC are well-established risk factors (28-30). Young age of onset and detection of MSI are typical for the development of SBA in the setting of HNPCC. Our data and the findings of two other studies $(31,32)$ identifying MSI in 67 and $73 \%$ of CD-associated carcinomas, respectively, show that these features also occur in $\mathrm{CD}$ associated SBAs. Therefore, CD should be included in the differential diagnosis of SBAs exhibiting MSI.

Aberrant methylation at $\mathrm{CpG}$ islands (CIM) is an epigenetic phenomenon found in cancer and non-neoplastic conditions including chronic inflammation and aging (14-16). By evaluating the methylation status in CD-associated carcinomas and non-neoplastic mucosa samples, we aimed to identify a molecular link between CD-associated inflammation and carcinogenesis. In line with a causative role of $\mathrm{CD}$ for aberrant methylation, CIM was identified in the majority of the non-neoplastic mucosa samples of the CD patients and in all of the CD-associated carcinomas investigated herein. While methylation at the MINT and MGMT loci was noted in both non-neoplastic and neoplastic tissues, MLH1 methylation and loss of MLH1 expression were exclusively found in carcinomas of the CD patients indicating MLH1 methylation to be the critical step in CD-associated carcinogenesis. CIM, however, was not found to be specific for CD. We detected low-level CIM in the majority of non-CD tissues investigated, 
indicating alternative causes of CIM. The average age of patients with CIM-negative and CIM-positive non-CD carcinomas was 55 and 73 years, respectively, supporting the hypothesis of aberrant methylation occurring in the course of aging (14). High-level CIM, methylation-induced silencing of $\mathrm{MLHI}$ and MSI were found in 2 non-CD carcinomas and were not specific for CD-associated SBAs. Detection of this pathway in all of the CD-associated SBAs in the present study, however, provide evidence for a link of this epigenetically initiated pathway to chronic inflammation.

In conclusion, we confirmed $\mathrm{CD}$ as a risk factor for SBA and further demonstrated the high-level CIM/MSI pathway to be typical of CD-associated small bowel carcinomas. Our findings suggest that $\mathrm{CD}$ should be considered in patients with small bowel adenocarcinoma, particularly when the tumors display MSI.

\section{Acknowledgements}

We thank Stefanie Kellner for the outstanding technical assistance.

\section{References}

1. Green PHR, Stavropoulos SN, Panagi SG, Goldstein SL, Mcmahon DJ, Absan H and Neugut AI: Characteristics of adult celiac disease in the USA: results of a national survey. Am J Gastroenterol 96: 126-131, 2001.

2. Green PH and Cellier C: Celiac disease. N Engl J Med 357: 1731-1743, 2007.

3. Marsh MN: The morphology and immunopathology of the jejunal lesion in gluten sensitivity. Eur J Gastroenterol Hepatol 3: 163-168, 1991.

4. Oberhuber G, Granditsch G and Vogelsang H: The histopathology of coeliac disease: time for a standardized report scheme for pathologists. Eur J Gastroenterol Hepatol 11: 11851194,1999

5. Catassi C, Bearzi I and Holmes GK: Association of celiac disease and intestinal lymphomas and other cancers. Gastroenterology 128: S79-S86, 2005.

6. Swinson CM, Slavin G, Coles EC and Booth CC: Coeliac disease and malignancy. Lancet 1: 111-115, 1983 .

7. Rampertab SD, Forde KA and Green PH: Small bowel neoplasia in coeliac disease. Gut 52: 1211-1214, 2003.

8. Askling J, Linet M, Gridley G, Halstensen TS, Ekström K and Ekbom A: Cancer incidence in a population-based cohort of individuals hospitalized with celiac disease or dermatitis herpetiformis. Gastroenterology 123: 1428-1435, 2002.

9. Howdle PD, Jalal PK, Holmes GK and Houlston RS: Primary small-bowel malignancy in the UK and its association with coeliac disease. QJM 96: 345-353, 2003.

10. Rewers M: Epidemiology of celiac disease: what are the prevalence, incidence, and progression of celiac disease? Gastroenterology 128: S47-S51, 2005.

11. Johnston SD and Watson RG: Small bowel lymphoma in unrecognized coeliac disease: a cause for concern? Eur J Gastroenterol Hepatol 12: 645-648, 2000.

12. Bariol C, Suter C, Cheong K, Ku SL, Meagher A, Hawkins N and Ward R: The relationship between hypomethylation and $\mathrm{CpG}$ island methylation in colorectal neoplasia. Am J Pathol 162: 1361-1371, 2003

13. Jass JR: Classification of colorectal cancer based on correlation of clinical, morphological and molecular features. Histopathology 50: $113-130,2007$

14. Waki T, Tamura G, Sato M and Motoyama T: Age-related methylation of tumor suppressor and tumor-related genes: an analysis of autopsy samples. Oncogene 22: 4128-4133, 2003.
15. Issa JP, Ahuja N, Toyota M, Bronner MP and Brentnall TA: Accelerated age-related $\mathrm{CpG}$ island methylation in ulcerative colitis. Cancer Res 61: 3573-3577, 2001.

16. Kang GH, Lee HJ, Hwang KS, Lee S, Kim JH and Kim JS: Aberrant CpG island hypermethylation of chronic gastritis, in relation to aging, gender, intestinal metaplasia, and chronic inflammation. Am J Pathol 163: 1551-1556, 2003.

17. Michel S, Kloor M, Singh S, Gdynia G, Roth W, von Knebel Doeberitz M, Schirmacher P and Bläker H: Coding microsatellite instability analysis in microsatellite unstable small intestinal adenocarcinomas identifies MARCKS as a common target of inactivation. Mol Carcinog 49: 175-182, 2010.

18. Boland CR, Thibodeau SN, Hamilton SR, Sidransky D, Eshleman JR, Burt RW, Meltzer SJ, Rodriguez-Bigas MA, Fodde R, Ranzani GN and Srivastava S: National Cancer Institute Workshop on Microsatellite Instability for cancer detection and familial predisposition: development of international criteria for the determination of microsatellite instability in colorectal cancer. Cancer Res 58: 5248-5257, 1998.

19. Findeisen P, Kloor M, Merx S, Sutter C, Woerner SM, Dostmann N, Benner A, Dondog B, Pawlita M, Dippold W, Wagner R, Gebert J and von Knebel Doeberitz M: T25 repeat in the 3' untranslated region of the CASP2 gene: a sensitive and specific marker for microsatellite instability in colorectal cancer. Cancer Res 65: 8072-8078, 2005.

20. Chan AO, Issa JP, Morris JS, Hamilton SR and Rashid A: Concordant $\mathrm{CpG}$ island methylation in hyperplastic polyposis. Am J Pathol 160: 529-536, 2002.

21. Esteller M, Hamilton SR, Burger PC, Baylin SB and Herman JG: Inactivation of the DNA repair gene O6-methylguanine-DNA methyltransferase by promoter hypermethylation is a common event in primary human neoplasia. Cancer Res 59: 793-797, 1999.

22. Herman JG, Graff JR, Myöhänen S, Nelkin BD and Baylin SB: Methylation-specific PCR: a novel PCR assay for methylation status of CpG islands. Proc Natl Acad Sci USA 93: 9821-9826, 1996.

23. Park SJ, Rashid A, Lee JH, Kim SG, Hamilton SR and Wu TT: Frequent $\mathrm{CpG}$ island methylation in serrated adenomas of the colorectum. Am J Pathol 162: 815-822, 2003.

24. West J, Logan RF, Hill PG, Lloyd A, Lewis S, Hubbard R, Reader R, Holmes GK and Khaw KT: Seroprevalence, correlates, and characteristics of undetected celiac disease in England. Gut 52: 960-965, 2003

25. Di Sabatino A and Corazza GR: Coeliac disease. Lancet 373: 1480-1493, 2009

26. Silano M, Volta U, Vincenzi AD, Dessì M and Vincenzi MD; Collaborating Centers of the Italian Registry of the Complications of Coeliac Disease: Effect of a gluten-free diet on the risk of enteropathy-associated T-cell lymphoma in celiac disease. Dig Dis Sci 53: 972-976, 2008.

27. Kingham JG, Ramanaden D and Dawson A: Metachronous small-bowel adenocarcinoma in coeliac disease: gluten-free diet is not protective. Scand J Gastroenterol 33: 218-222, 1998.

28. Piton G, Cosnes J, Monnet E, Beaugerie L, Seksik P, Savoye G, Cadiot G, Flourie B, Capelle P, Marteau P, Lemann M, Colombel JF, Khouri E, Bonaz B and Carbonnel F: Risk factors associated with small bowel adenocarcinoma in Crohn's disease: a case-control study. Am J Gastroenterol 103: 1730-1736, 2008.

29. Lynch HT, Smyrk TC, Lynch PM, Lanspa SJ, Boman BM, Ens J, Lynch JF, Strayhorn P, Carmody T and Cristofaro G: Adenocarcinoma of the small bowel in Lynch syndrome II. Cancer 64: 2178-2183, 1989.

30. Fearnhead NS, Britton MP and Bodmer WF: The ABC of APC. Hum Mol Genet 10: 721-733, 2001.

31. Potter DD, Murray JA, Donohue JH, Burgart LJ, Nagorney DM, van Heerden JA, Plevak MF, Zinsmeister AR and Thibodeau SN: The role of defective mismatch repair in small bowel adenocarcinoma in celiac disease. Cancer Res 64: 7073-7077, 2004.

32. Diosdado B, Buffart TE, Watkins R, Carvalho B, Ylstra B, Tijssen M, Bolijn AS, Lewis F, Maude K, Verbeke C, Nagtegaal ID, Grabsch H, Mulder CJ, Quirke P, Howdle P and Meijer GA: High-resolution array comparative genomic hybridization in sporadic and celiac disease-related small bowel adenocarcinomas. Clin Cancer Res 16: 1391-1401, 2010. 